\title{
DNA Sequence Dimorphisms in Populations of the Clubroot Pathogen Plasmodiophora brassicae
}

Yalong Yang and Krista Zuzak, Alberta Plant Health Lab, Crop Diversification Centre North, Alberta Agriculture and Forestry, Edmonton, AB, T5Y 6H3, Canada; Michael Harding, Crop Diversification Centre South, Alberta Agriculture and Forestry, Brooks, AB, T1R 1E6, Canada; Stephen Strelkov, Department of Agricultural, Food and Nutritional Science, University of Alberta, Edmonton, AB T6G 2P5, Canada; Sheau-Fang Hwang, David Feindel, Crop Diversification Centre North, Alberta Agriculture and Forestry, Edmonton, AB, T5Y 6H3, Canada; and Jie Feng, ${ }^{\dagger}$ Alberta Plant Health Lab, Crop Diversification Centre North, Alberta Agriculture and Forestry

\begin{abstract}
To develop genetic markers for differentiation between pathotypes of the clubroot pathogen Plasmodiophora brassicae, DNA polymorphisms of $85 P$. brassicae genes were investigated by comparing the sequences of these genes from published expressed sequence tag libraries to their sequences in the two released whole genomes. A significant portion of the identified sequence differences across all polymorphic genes are between an isolate from New Zealand and the two whole-genome sequenced isolates. Four genes with a high density of polymorphisms were selected and their partial sequences were amplified by polymerase chain reaction

(PCR) from the old pathotypes 2, 3, 5, 6, and 8 (based on the Williams differential set) and the new virulent populations 3-like and 5-like. On the sequences of two of the four genes, the old pathotypes are all identical to the two whole-genome sequenced isolates and all of the new virulent populations are identical to the New Zealand isolate. Based on the dimorphism on the sequence of these two genes, an RNase H-dependent PCR protocol was developed. This protocol was demonstrated to be useful for virulent pathotype identification and may also be used to study the population dynamics of $P$. brassicae and the in planta interaction of different pathotypes.
\end{abstract}

Clubroot, caused by the protist Plasmodiophora brassicae Woronin, is an important threat to Canadian canola (Brassica napus) production (Hwang et al. 2012). In the Canadian Prairies, clubroot was first identified on canola in 2003, in a dozen fields near Edmonton, Alberta (Tewari et al. 2005). The disease has since then spread throughout central Alberta and has also been confirmed in canola fields in Saskatchewan and Manitoba (Strelkov and Hwang 2014).

Many strategies have been proposed for clubroot control (Peng et al. 2014) and, among those, breeding for resistance is believed to be most effective (Rahman et al. 2014). Several clubroot-resistant canola hybrids have been bred and introduced into the Canadian market (Strelkov and Hwang 2014). However, clubroot resistance can erode quickly in response to the selection pressure imposed by repeated cropping of a resistant cultivar (Strelkov et al. 2016). A province-wide survey for clubroot conducted in 2013 found six fields of resistant canola in Alberta in which disease severity was greater than expected for clubroot-resistant hosts (Strelkov et al. 2014). On the Williams differential hosts (Williams 1966), these field populations displayed the virulence profile of pathotype 5 but, unlike the original pathotype 5 populations, they were virulent on all registered resistant cultivars (Strelkov et al. 2016). It was apparent that the Williams set of differential hosts did not have sufficient resolution to discriminate pathotype 5 from this virulent pathotype. As a result, this new pathotype was temporarily named pathotype $5 x$. In 2016, significant clubroot symptoms were found on clubroot-resistant canola cultivars in more than 40 fields identified in a follow-up survey (Strelkov et al. 2017). Testing of the 2016 populations on the Williams differentials indicated that the retrieved isolates were not limited to the pathotype 5 virulence pattern but also included patterns characteristic of other pathotypes (Strelkov et al. 2016) (J. Feng, unpublished).

${ }^{\dagger}$ Corresponding author: J. Feng; E-mail: jie.feng@gov.ab.ca

Funding: This work was supported by the Alberta Crop Industry Development Fund (grant number 2012C015D).

Accepted for publication 5 April 2018.

@ 2018 The American Phytopathological Society
With the detection of new virulent pathotypes not controlled by existing clubroot resistance in canola, the ability to rapidly differentiate pathotypes and identify new pathotypes has become extremely important for diagnosis of control failures and development of control strategies and breeding for effective resistance. To discover and develop genetic markers for pathotype differentiation, DNA polymorphisms on $85 P$. brassicae genes were investigated by comparing the sequences of the 85 expressed sequence tags (EST) to their corresponding sequences in the two released whole genomes. Based on the sequences of two identified polymorphic genes, an RNase H-dependent polymerase chain reaction (rhPCR) protocol was developed. This protocol can be used to differentiate between two classes of $P$. brassicae pathotypes; namely, new virulent pathotypes from the old pathotypes.

\section{Materials and Methods}

$\boldsymbol{P}$. brassicae populations. Terminologies for population, singlespore isolate, and pathotype were adopted from Xue et al. (2008). In this study, pathotypes characterized before the breakdown of resistance were defined as "old pathotypes" and populations virulent on resistant cultivars were defined as "new virulent populations" or "new virulent pathotypes". Seven populations of $P$. brassicae were provided by Dr. S. F. Hwang at the Crop Diversification Centre North $(\mathrm{CDCN})$. These populations included five single-spore isolates (SACAN-ss3, SACAN-ss1, ORCA-ss4, AbotJE-ss1, and CDCNss3), which are classified as pathotypes 2, 3, 5, 6, and 8, respectively, on Williams deferential hosts (Williams 1966; Xue et al. 2008), and two populations that could be classified as pathotype 3 or 5 , respectively, on Williams deferential hosts but are virulent to the resistant gene carried by the resistant canola cultivar $45 \mathrm{H} 29$. The latter two populations had been selected on $45 \mathrm{H} 29$ for many generations in greenhouses and were named 3-like and 5-like, respectively, in this study. In addition, four populations were represented by field galls (namely, G1, G2, G3, and G4), which are similar to the 5-like population but have not been repeatedly selected on $45 \mathrm{H} 29$ or against other resistance genes. To initiate the present study, all of the above 11 populations were regenerated on canola in the Alberta Plant Health Lab, CDCN with the 5 single-spore isolates on the susceptible cultivar Westar; G1, G2, G3, and G4 on 45H29; and 3-like and 5-like on both Westar and $45 \mathrm{H} 29$ (Table 1). Thus, in total, 13 populations from Alberta (Table 1) were prepared and utilized in this study.

General molecular techniques. Extraction of genomic DNA was performed using the DNeasy Plant Mini kit (Qiagen Canada, 
Toronto, ON, Canada). Primers (Table 2) for PCR were synthesized by Integrated DNA Technologies (Coralville, IA). All PCR were conducted in Promega PCR master mix (Promega Corp., Madison, WI) using a ProFlex $3 \times 32-W e l l$ PCR system (Fisher Scientific Canada, Ottawa. ON, Canada). Purification of PCR product was performed using the Wizard SV Gel and PCR Cleanup System (Promega Corp.). Electrophoresis was performed on 1\% agarose gels with GelGreen (VWR Canada, Mississauga, ON, Canada) staining. DNA bands were visualized using a GelDoc EZ Imaging System (Bio-Rad Canada, Mississauga, ON, Canada). DNA sequencing was conducted by the Department of Biological Sciences, University of Alberta (Edmonton, AB, Canada). The chromatograms of sequencing files were viewed by the BioEdit software (Hall 1999).

Gene screening. Sequences of 85 P. brassicae genes, represented by EST, were retrieved from the National Center for Biotechnology Information (NCBI) database by searching their GenBank accession numbers, available in Bulman et al. (2006, 2007). All of these genes were derived from a New Zealand isolate, named CNZ in this study. The genomic sequences of these 85 genes in the two whole-genome sequenced $P$. brassicae isolates, e3 from Germany (unknown pathotype) (Fähling et al. 2003; Schwelm et al. 2015) and ZJ-1 from China (pathotype 1 on the Williams differential set) (Bi et al. 2016), were obtained by BlastN using these 85 genes as a batch query against the NCBI whole-genome shotgun contigs organism $P$. brassicae (Tax ID 37360), a database containing only the whole-genome sequences of e 3 and $\mathrm{ZJ}-1$ when this study was conducted (last verified 4 February 2018). For each of these 85 genes, the EST sequence in $\mathrm{CNZ}$ and genomic sequences in e 3 and ZJ-1 were compared by alignment using Clustal Omega (https://www.ebi.ac.uk/Tools/msa/ clustalo). PCR primers (Table 2) were designed for four genes that have a high density of nucleotide polymorphisms among the three isolates.

Table 1. Plasmodiophora brassicae populations from which the DNA sequences used in this study were derived

\begin{tabular}{llll}
\hline Group $^{\mathbf{a}}$ & \multicolumn{1}{c}{ Populations } & \multicolumn{1}{c}{ Description $^{\mathbf{b}}$} & \multicolumn{1}{c}{ Origin } \\
\hline $\mathrm{X}$ & Pathotype 2, 3,5,6 and 8 & SSI; galls on Westar & Alberta \\
& e3 & SSI, WGS & Germany $^{\mathrm{c}}$ \\
& ZJ-1 & SSI, WGS & China $^{\mathrm{d}}$ \\
$\mathrm{Y}$ & CNZ & SSI & New Zealand \\
$\mathrm{Z}$ & 3-like R & Galls on 45H29 & Alberta \\
& 3-like S & Galls on Westar & Alberta \\
& 5-like R & Galls on 45H29 & Alberta \\
& 5-like S & Galls on Westar & Alberta \\
& G1, G2, G3, and G4 & Galls on 45H29 & Alberta \\
\hline
\end{tabular}

${ }^{a}$ Grouped in the present study.

${ }^{\mathrm{b}} \mathrm{SSI}=$ single-spore isolate and WGS $=$ whole genome sequenced.

${ }^{\mathrm{c}}$ References: Fähling et al. 2003 and Schwelm et al. 2015.

${ }^{\mathrm{d}}$ Reference: Bi et al. 2016.

e References: Bulman et al. 2006, 2007.
PCR amplification of gene fragments. Resting spores were prepared from galls of the 13 populations following the protocol described by Feng et al. (2012a). Genomic DNA was extracted from the prepared resting spores. Against each of these DNA samples, a fragment of each of the four selected genes was amplified by PCR using primers $\mathrm{P}-1, \mathrm{P}-2, \mathrm{P}-3$, and P-4 (Table 2); with 35 cycles of denaturation at $94^{\circ} \mathrm{C}$ for $30 \mathrm{~s} \mathrm{(3} \mathrm{min} \mathrm{for} \mathrm{the} \mathrm{initial} \mathrm{denaturation),} \mathrm{anneal-}$ ing at $47^{\circ} \mathrm{C}$ (primer pairs $\mathrm{P}-2$ and $\mathrm{P}-3$ ) or $50^{\circ} \mathrm{C}$ (primer pairs $\mathrm{P}-1$ and $\mathrm{P}-4$ ) for $45 \mathrm{~s}$, and extension at $72^{\circ} \mathrm{C}$ for $1 \mathrm{~min}$; and a final extension at $72^{\circ} \mathrm{C}$ for $5 \mathrm{~min}$. All PCR products were purified and sequenced from both directions using the forward and reverse PCR primers.

rhPCR. For each of the four selected genes, the sequenced PCR products from the 13 P. brassicae populations were compared using multiple alignment. Based on the aligned sequences of two genes, two pairs of rhPCR primers, with one for one gene, were manually designed according to Dobosy et al. (2011). Duplex rhPCR was conducted using these two primer pairs on the DNA sample extracted from each of the 13 populations. Each $15-\mu l$ reaction contained 1.5 $\mathrm{mU}$ of RNase $\mathrm{H} 2$ (Integrated DNA Technologies), $0.25 \mu \mathrm{M}$ each of the four primers, and $50 \mathrm{ng}$ of template DNA. The rhPCR program

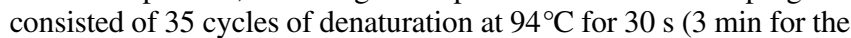
initial denaturation), annealing at $62^{\circ} \mathrm{C}$ for $45 \mathrm{~s}$; and extension at $72^{\circ} \mathrm{C}$ for $1 \mathrm{~min}$; and a final extension at $72^{\circ} \mathrm{C}$ for $5 \mathrm{~min}$. After gel electrophoresis, the upper bands produced by G1, G2, G3, and G4 in the rhPCR products were sequenced with the primer $5^{\prime}$ GCTGGGTGCCGCTATACC-3' (Fig. 1). In addition, the potential use of rhPCR in quantification of the biomass of different $P$. brassicae pathotypes was evaluated by running the duplex rhPCR against DNA samples consisting of serial dilutions of DNA from pathotype 3 and the 3-like population. The ingredients (except template) of the rhPCR were same as described above. The rhPCR program was also the same as above, except that the cycle number was changed to 30 .

\section{Results}

All 85 genes identified from CNZ are present in the whole-genome sequences of e3. In contrast, 83 are present in the whole-genome sequences of ZJ-1, with AM180201 and AM177649 absent. In total, 79 genes are single-copied in e3 and 72 are single-copied in both e 3 and ZJ-1. Among these 72 genes, 56 carry polymorphisms on the coding sequences as defined by the length of the EST among the three isolates. Among these 56 genes, 42 have identical sequences in e 3 and ZJ-1. From these 42 genes, AM180213, AM180230, AM411654, and AM411657 were selected for further studies. When these four genes in different Alberta $P$. brassicae populations were sequenced and the obtained sequences were compared with each other and with those in CNZ, e3, and ZJ-1, dimorphisms were found on the sequences of AM411654 and AM411657, in which pathotypes 2, 3, 5, 6 , and 8 , and isolates e 3 and $\mathrm{ZJ}-1$ (group $\mathrm{X}$ in Table 1) shared one sequence and all other tested populations (groups $\mathrm{Y}$ and $\mathrm{Z}$ in Table 1) shared another sequence. The dimorphisms of the two genes are illustrated in Figure 1. In addition, dimorphisms were also found in

Table 2. Polymerase chain reaction primers used in this study

\begin{tabular}{|c|c|c|c|}
\hline Primer pair & $\begin{array}{l}\text { Sequences of primers, forward (up) } \\
\text { and reverse (down) }\end{array}$ & GenBank accession number & Amplicon (bp) $)^{\mathbf{a}}$ \\
\hline \multirow[t]{2}{*}{$\mathrm{P}-1$} & AAGAAGACGTACTCCACGAAC & AM180213 & 214 \\
\hline & TCCGGTGTTAGAGTATGGGAC & $\ldots$ & $\ldots$ \\
\hline \multirow[t]{2}{*}{$\mathrm{P}-2$} & AGCTTGTCAAGGCGATGATTG & AM180230 & 298 \\
\hline & ACGCAYACTGTGTATCAGATG & $\ldots$ & $\ldots$ \\
\hline \multirow[t]{2}{*}{$\mathrm{P}-3$} & ATGTCAGATCGTCAACGGTAC & AM411654 & 490 \\
\hline & CGAAAGAAGAAGTAGTCGCAG & $\ldots$ & $\ldots$ \\
\hline \multirow[t]{2}{*}{$\mathrm{rhP}-3$} & GCTGGGTGCCGCTATACCrGACCCC/3SpC3/ & AM411654 & 337 \\
\hline & GTCGCAGGATGGGTCCCArCTCGCC/3SpC3/ & $\ldots$ & $\ldots$ \\
\hline \multirow[t]{2}{*}{$\mathrm{P}-4$} & ACATCGCCTGTTTCACGGATTG & AM411657 & 467 \\
\hline & TGAGGTACAAGAAGTCGGCATC & $\ldots$ & $\ldots$ \\
\hline \multirow[t]{2}{*}{ rhP-4 } & AGCTGGTCCAGAGTGCACrCCACGG/3SpC3/ & AM411657 & 135 \\
\hline & AAGCGCCCACCCTCTTGrGAGTGG/3SpC3/ & $\ldots$ & $\ldots$ \\
\hline
\end{tabular}

${ }^{a}$ Size of genomic DNA in isolate e 3 embraced by the primer pair. 


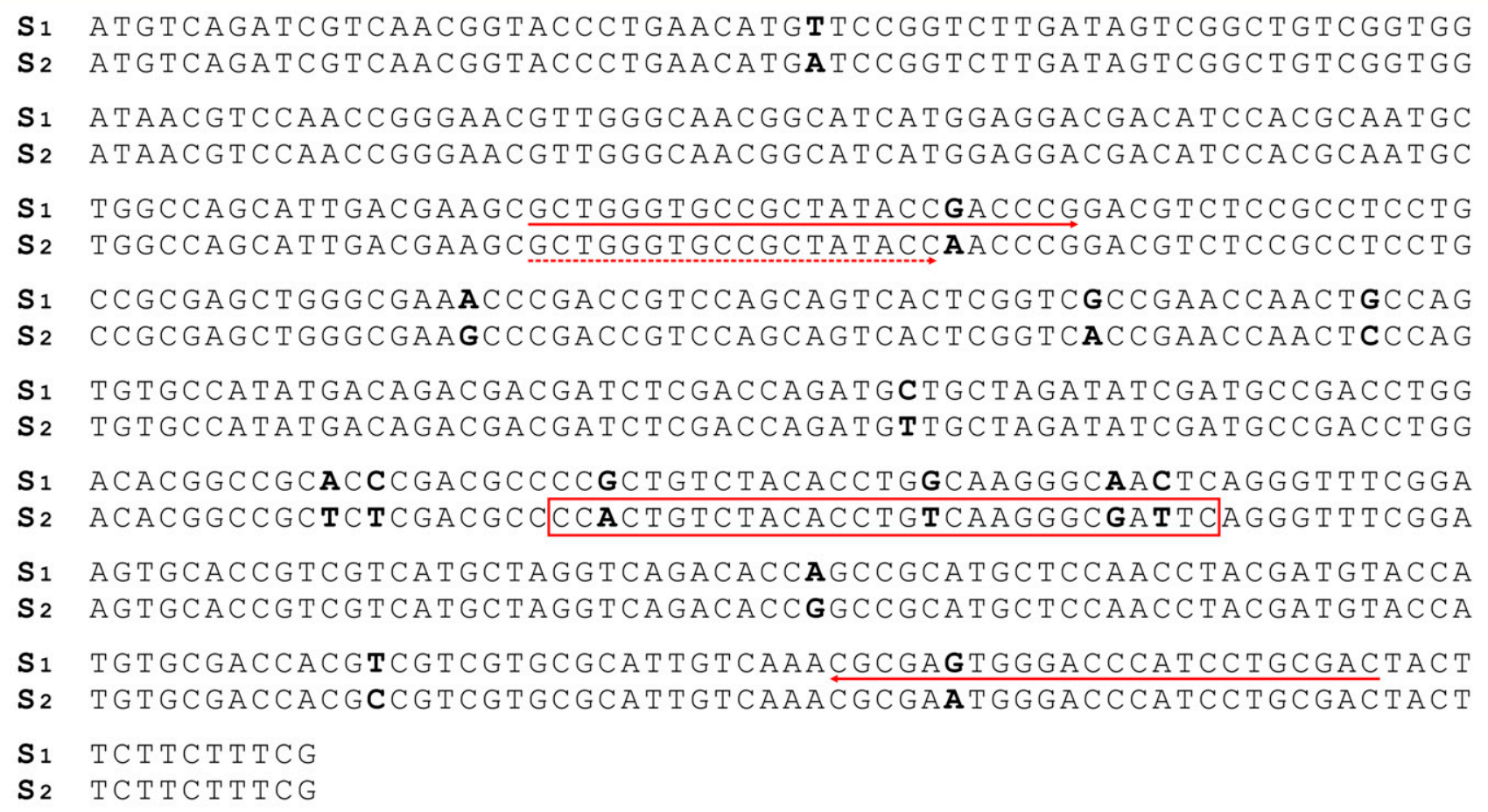

S1 ACATCGCCTGTTTCACGGATTGGTACCA GCGCGTGCACATCGGCA GG G GAATGCAAACA $\mathbf{S}_{3}$ ACATCGCCTGTT TCACGGATTGGTACCAGCGCGTGCACATCGGCAGGGCGAATGCAAACA $\mathrm{S}_{4}$ ACATCGCCTGTT TCACGGATTGGTACCA GCGCGTGCACATCGGCAGGGCGAATGCAAACA

S1 AGTGGATTACAATCGGGGGATCATACCCCGGCGCTCTGGCAGCATGGTCTGCGGTCCATC S 3 AGTGGATTACAATCGGAGGATCATACCCCGGCGCTCTGGCAGCATG - - - - - - - - - - - S4 AGTGGATTACAATCGGAGGATCATACCCCGGCGCTCTGGCAGCATGGTCTGCGGTCCATC

S1 GGCTCTCTGCCCAATGGTCAGACTCTGAATTCGAATCGTCAGGTATCGCCTGAAGTACCC

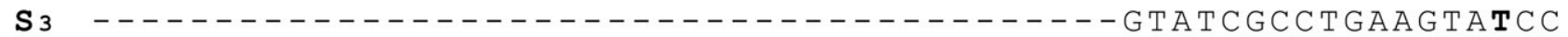
$\mathbf{S}_{4}$ GGCTCTCTGCCCAATGGACAGACTCTGAATTCAATCGTCAGGTATCGCCTGAAGTATCC

S1 GCATCTGACAGCAGGCGCACTGGCATCGAGCGCTGTGGTTGCCCCGTTTGCAGAGT TCCC S 3 GCATCTGACAGCAGGCGCGCTGGCATCGAGCGCTGTGGTTGCCCCGTTTGCAGAGTTCCC S 4 GCATCTGACA CCAGGCGCGCTGGCATCGAGCGCTGTGGTTGCCCCGT T TGCAGAGT TCCC

S1 TGAATCGACGAGCAAGTGGCATCATCAGCTGGTCCAGCGTGCACACACGCGCTGCAAGA $\mathbf{S}_{3}$ TGAATTCGACGAACAAGTGGCATTATCAGCTGGTCCAGAGTGCACCCACGCGCTGCAAGA S4 TGAAT TCGACGAACAAGTGGCATTATCAGCTGGTCCAGAGTGCACCCACGCGCTGCAAGA

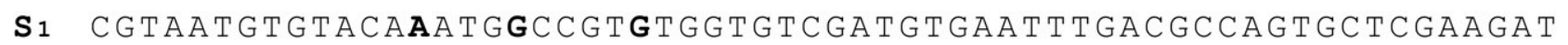

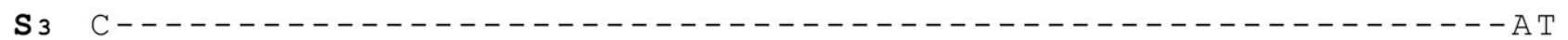
S4 CGTAATTGTACAGATGTCCGTCTGGTGTCGATGTGAATTTGACGCCAGTGCTCGAAGAT

S1 CACTGCAATGGTGGAAGGTGCACT TCAAGAGGGTGGGCGCTTGGCTGACGAAATGAAGGC S 3 CACTGCAATGGTGGAAGGTGCAC TCCAAGA GG T G GCGCT T GGCTGACGAAATGAAGGC

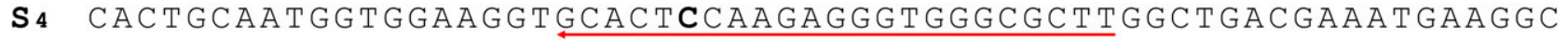

S1 CCTGTTTCGTGCTCGCAATtTCGGATGCGACTTCTTGACCTCA S 3 CCTGTTTCGTGCTCGCAATTGTCGATGCCGACTTCTTGTACCTCA S4 CCTGTTTCGTGCTCGCAATTGTGGATGCCGATTCTTGTACCTCA

Fig. 1. DNA sequence dimorphism of GenBank accession numbers AM411654 (up) and AM411657 (down) among Plasmodiophora brassicae populations. $\mathrm{S}_{1}, \mathrm{~S}_{2}, \mathrm{~S}_{3}$, and $\mathrm{S}_{4}$, populations correspondent to the groups X (pathotypes 2, 3, 5, 6, and 8, and isolates e3 and ZJ-1), Y (CNZ), and Z (3-like R, 3-like S, 5-like R, 5-like S, G1, G2, G3, and G4), with $S_{1}=X, S_{2}=Y+Z, S_{3}=Y$, and $S_{4}=Z$. Dimorphic nucleotides are shown in bold. The solid arrow lines indicate the RNase H-dependent polymerase chain reaction (rhPCR) primers and the dotted arrow line indicates the sequencing primer for the upper bands produced by G1, G2, G3, and G4. From a DNA sample derived from a mixture of $\mathrm{S}_{1}$ and $\mathrm{S}_{2}$ populations, the rhPCR primer pair for AM411654 amplified a fragment of $337 \mathrm{bp}$ from the $\mathrm{S}_{1}$ populations only and the primer pair for AM411657 amplified a fragment of 135 bp from the $S_{2}$ (i.e., $S_{3}$ and $S_{4}$ ) populations only. A 29-bp fragment is boxed in the upper panel. 
AM180213 and AM180230, in which pathotypes 2, 3, 5, 6, and 8, and $\mathrm{CNZ}$ shared one sequence and all other tested populations shared another sequence (data not shown). Among the four dimorphic genes, AM411657 was the only one that carried introns on its genomic sequences defined by the primer pair P-4 (Table 2). Thus, the genomic sequence of AM411657 in populations belonging to groups $Y$ and $\mathrm{Z}$ (Table 1) represented a new sequence and, therefore, was submitted to GenBank with accession number MH084665.

Two rhPCR primer pairs specific to AM411654 and AM411657, respectively, were designed (Fig. 1; Table 2). The primer pair rhP-3 amplified a band at $337 \mathrm{bp}$ from populations belonging to group $\mathrm{X}$ but not groups $\mathrm{Y}$ and $\mathrm{Z}$, whereas the primer pair rhP-4 amplified a band at 135 bp from populations belonging to groups $\mathrm{Y}$ and $\mathrm{Z}$ but not group X. Duplex rhPCR using the two primer pairs amplified the 337-bp band from pathotypes 2, 3, 5, 6, and 8, and the 135-bp band from the two 3-like and two 5-like populations (Fig. 2A). The four field populations (G1 to G4) produced both bands, suggesting that they were mixtures of different isolates, which was not consistent with the sequencing results (Fig. 2B). The 337-bp bands from these four populations were purified and sequenced. The resultant sequences are identical to each other and to the corresponding sequence in the populations belonging to group X.

When mixtures of pathotype 3 and 3-like DNA were tested with the duplex rhPCR, both the 337- and 135-bp bands were obtained (Fig. 3). The 337-bp band became stronger when the proportion of pathotype 3 DNA in the DNA sample increased and, similarly, the 135-bp band became stronger with the increased proportion of the 3-like DNA (Fig. 3), indicating the potential usage of this duplex rhPCR technique in evaluating the purity of $P$. brassicae populations.

\section{Discussion}

Sequence polymorphisms were found between a high proportion of genes among the $P$. brassicae isolates e $3, \mathrm{ZJ}-1$, and CNZ. Interestingly, the sequences of most of these genes in e 3 and $\mathrm{ZJ}-1$ are identical, suggesting that the polymorphisms are likely dimorphisms. When the four selected genes in Alberta P. brassicae populations were sequenced, the dimorphic nature of the polymorphisms was confirmed. For all four genes, the new virulent populations share one sequence and the old pathotypes share the other sequence. These dimorphisms suggest that the new virulent pathotypes and the old pathotypes may belong to different lineages. The extensiveness of the polymorphisms across the tested genes also suggests that the new virulent pathotypes might have gone through a lengthy

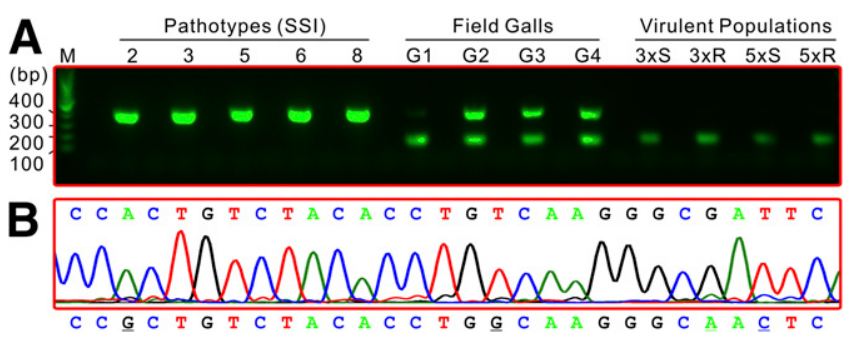

Fig. 2. Differentiation of Plasmodiophora brassicae populations by analyzing the dimorphic sequences of two selected genes. A, RNase H-dependent polymerase chain reaction (PCR) assay. Lanes $2,3,5,6$, and $8=$ single-spore isolates (SSI). Lanes $\mathrm{G} 1, \mathrm{G} 2, \mathrm{G} 3$, and $\mathrm{G} 4$ = four field populations that are virulent on the resistant canola $45 \mathrm{H} 29$. Lane $\mathrm{M}=$ Promega 100-bp DNA ladder. Lanes $3 \times \mathrm{S}$ and $3 \times \mathrm{R}=$ the 3 -like populations on the susceptible canola Westar and $45 \mathrm{H} 29$, respectively, and lanes $5 \times S$ and $5 \times R=$ the 5 -like populations on Westar and $45 \mathrm{H} 29$, respectively. $B$, Sequencing chromatogram of a 29-bp fragment from population $\mathrm{G} 2$, which illustrates that the RNase H-dependent PCR could but sequencing of PCR products could not identify the contamination of new virulent populations by old populations. The PCR product was amplified by primer pair P-3 and sequenced using the forward primer of P-3. The resultant sequence after sequencing is listed on the top, which is identical to the sequences of the corresponding fragments in isolate CNZ and the new virulent isolates. The sequence of the corresponding fragments in isolates e 3 and $\mathrm{ZJ}-1$ and the old pathotypes is listed at the bottom, with the dimorphic nucleotides underlined. coevolution with the hosts, and their emergence in Alberta was not simply because of the loss of an avirulence gene or mutations on a few genes.

The rhPCR technique relies on primers specific to the DNA sequences carrying the single nucleotide polymorphism between individuals (Dobosy et al. 2011). In this study, the duplex rhPCR was able to differentiate new virulent pathotypes from the old avirulent pathotypes. However, this was only limited to the populations accessible by the Alberta Plant Health Lab. We did not test more field populations in addition to $\mathrm{G} 1, \mathrm{G} 2, \mathrm{G} 3$, and $\mathrm{G} 4$, partially due to our speculation that many field populations with a history of canola production were likely composed of both old and new pathotypes. In these circumstances, it would be difficult to use molecular markers for pathotype differentiation on field galls. Thus, based on the data obtained from the present study, we would not recommend using the rhPCR protocol for molecular pathotyping of field populations before repeated selection on resistant hosts or the achievement of single-spore isolation.

Duplex rhPCR produced two bands from the populations G1, G2, G3, and G4. This is because each of the four populations was likely a mixture of new virulent pathotypes with a small proportion of old pathotypes. In such a mixture, the majority could overwhelm the minority in a sequencing reaction but could not do so in an rhPCR. For example, duplex rhPCR detected pathotype 3 from a template consisting of $1 \%$ pathotype 3 DNA and $99 \% 3$-like DNA. From such a mixture, sequencing may not be able to make the same detection. Therefore, the duplex rhPCR developed from the present study could be used to verify the purity of new virulent populations, especially if the population is represented by the original galls collected from the field.

The importance of determining the purity of populations was also emphasized by other studies. Jones (1980, cited after Dixon 2009) reported that more than one pathotype occurred within a spore suspension prepared from a single gall. Isolates of both old and new virulent pathotypes were obtained by single-spore isolation from single galls of both susceptible and resistant canola cultivars (S. E. Strelkov, unpublished). The coexistence of old and new virulent pathotypes, especially the persistence of old pathotypes in galls of resistant plants caused primarily by the new virulent pathotypes, suggested that there may be collaboration between the two pathotypes. Previous microscopic studies observed primary infection (root hair infection) on resistant (Diederichsen et al. 2009) and nonhost plants (Feng et al. $2012 \mathrm{~b}$ ). This infection might be caused by the virulent individuals coexisting with the avirulent population. Another possibility is that, as proposed by Feng et al. (2013), the primary infection of $P$. brassicae is indeed unspecific and the pathogenicity of a $P$. brassicae population is only determined in the postprimary infection stage. Studies on the interaction between a virulent pathotype and an avirulent pathotype during the coinfection process would provide important information on our understanding of clubroot pathogenesis and $P$. brassicae population dynamics. However, such studies have been difficult, due to the absence of a reliable method for pathotype purification and differentiation. The rhPCR protocols developed in this study, for the first time, provide a tool for the differentiation between the tested pathotypes. More importantly, the same rhPCR principle can be used in quantitative PCR (qPCR). Nucleotide dimorphisms present on all four genes investigated in this study (and likely on other

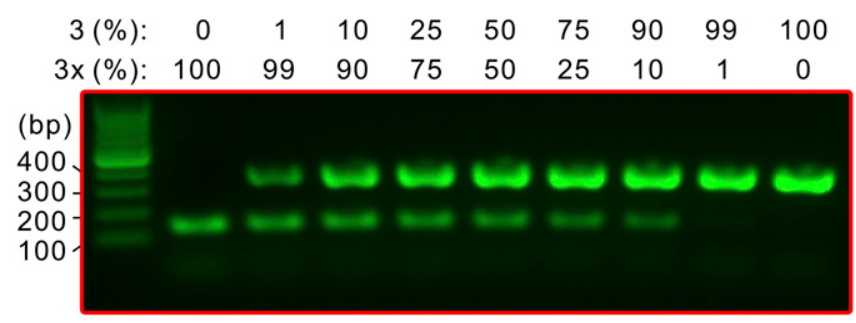

Fig. 3. RNase H-dependent polymerase chain reaction assay against DNA mixtures Row 3 = DNA from the isolate of pathotype 3 and row $3 x=$ DNA from the 3 -like population. Proportions of the two DNA for each reaction are listed on the top. The left-most line is the Promega 100-bp DNA ladder. 
polymorphic genes) allowed the design of rhPCR primers that can be used for qPCR analyses. Currently, two quantitative rhPCR primer pairs, one specific to pathotype 5 and the other one to the 5-like population, are being evaluated in our lab for their usefulness on biomass quantification from canola roots infected by a mixture of the two $P$. brassicae populations. By a time-course analysis, information on the dynamics of a virulent population and an avirulent population during pathogenesis will be revealed for the first time.

\section{Literature Cited}

Bi, K., He, Z., Gao, Z., Zhao, Y., Fu, Y., Cheng, J., Xie, J., Jiang, D., and Chen, T. 2016. Integrated omics study of lipid droplets from Plasmodiophora brassicae. Sci. Rep. 6: Article 36965.

Bulman, S. R., Ridgway, H. J., Eady, C., and Conner, A. J. 2007. Intron-rich gene structure in the intracellular plant parasite Plasmodiophora brassicae. Protist 158:423-433.

Bulman, S. R., Siemens, J., Ridgway, H. J., Eady, C., and Conner, A. J. 2006. Identification of genes from the obligate intracellular plant pathogen, Plasmodiophora brassicae. FEMS Microbiol. Lett. 264:198-204.

Diederichsen, E., Frauen, M., Linders, E. G. A., Hatakeyama, K., and Hirai, M. 2009. Status and perspectives of clubroot resistance breeding in crucifer crops. J. Plant Growth Regul. 28:265-281.

Dixon, G. R. 2009. Plasmodiophora brassicae in its environment. J. Plant Growth Regul. 28:212-228.

Dobosy, J. R., Rose, S. D., Beltz, K. R., Rupp, S. M., Powers, K. M., Behlke, M. A., and Walder, J. A. 2011. RNase H-dependent PCR (rhPCR): Improved specificity and single nucleotide polymorphism detection using blocked cleavable primers. BMC Biotechnol. 11:80.

Fähling, M., Graf, H., and Siemens, J. 2003. Pathotype separation of Plasmodiophora brassicae by the host plant. J. Phytopathol. 151:425-430.

Feng, J., Hwang, S. F., and Strelkov, S. E. 2012a. Analysis of expressed sequence tags derived from a compatible Plasmodiophora brassicae-canola interaction. Can. J. Plant Pathol. 34:562-574.

Feng, J., Hwang, S. F., and Strelkov, S. E. 2013. Studies into primary and secondary infection processes by Plasmodiophora brassicae on canola. Plant Pathol. 62:177-183.

Feng, J., Xiao, Q., Hwang, S. F., Strelkov, S. E., and Gossen, B. D. 2012 b. Infection of canola by secondary zoospores of Plasmodiophora brassicae produced on a nonhost. Eur. J. Plant Pathol. 132:309-315.
Hall, T. A. 1999. BioEdit: A user-friendly biological sequence alignment editor and analysis program for Windows 95/98/NT. Nucleic Acids Symp. Ser. Oxf. 41:95-98.

Hwang, S. F., Strelkov, S. E., Feng, J., Gossen, B. D., and Howard, R. J. 2012. Plasmodiophora brassicae: A review of an emerging pathogen of the Canadian canola (Brassica napus) crop. Mol. Plant Pathol. 13:105-113.

Jones, D. R. 1980. Differential pathogenicity of Plasmodiophora brassicae Wor. Ph.D. thesis, University of Cambridge.

Peng, G., Lahlali, R., Hwang, S. F., Pageau, D., Hynes, R. K., McDonald, M. R. Gossen, B. D., and Strelkov, S. E. 2014. Crop rotation, cultivar resistance, and fungicides/biofungicides for managing clubroot (Plasmodiophora brassicae) on canola. Can. J. Plant Pathol. 36:99-112.

Rahman, H., Peng, G., Yu, F., Falk, K. C., Kulkarni, M., and Selvaraj, G. 2014 Genetics and breeding for clubroot resistance in Canadian spring canola (Brassica napus L.). Can. J. Plant Pathol. 36:122-134.

Schwelm, A., Fogelqvist, J., Knaust, A., Jülke, S., Lilja, T., Bonilla-Rosso, G., Karlsson, M., Shevchenko, A., Dhandapani, V., Choi, S. R., Kim, H. G., Park, J. Y., Lim, Y. P., Ludwig-Müller, J., and Dixelius, C. 2015. The Plasmodiophora brassicae genome reveals insights in its life cycle and ancestry of chitin synthases. Sci. Rep. 5: Article 11153.

Strelkov, S. E., Manolii, V. P., Harding, M. W., Hwang, S. F., Manolii, E., Zuzak, K., Rennie, D. C., Feng, J., Rahaman, M., Daniels, G. C., Burke, D. A., Hill, T. B., Zahr, K., and Feindel, D. 2017. Occurrence and spread of clubroot on canola in Alberta in 2016. Can. Plant Dis. Surv. 97:164-167.

Strelkov, S. E., and Hwang, S. F. 2014. Clubroot in the Canadian canola crop: 10 years into the outbreak. Can. J. Plant Pathol. 36:27-36.

Strelkov, S. E., Hwang, S. F., Manolii, V. P., Cao, T., and Feindel, D. 2016 Emergence of new virulence phenotypes of Plasmodiophora brassicae on canola (Brassica napus) in Alberta, Canada. Eur. J. Plant Pathol. 145: 517-529.

Strelkov, S. E., Manolii, V. P., Harding, M. W., Hwang, S. F., Poscente, N., Lisowski, S. L. I., Pugh, C. A., and Burke, D. A. 2014. The occurrence of clubroot on canola in Alberta in 2013. Can. Plant Dis. Surv. 94:158-161.

Tewari, J. P., Strelkov, S. E., Orchard, D., Hartman, M., Lange, R. M., and Turkington, T. K. 2005. Identification of clubroot of crucifers on canola (Brassica napus) in Alberta. Can. J. Plant Pathol. 27:143-144.

Williams, P. H. 1966. A system for the determination of races of Plasmodiophora brassicae that infect cabbage and rutabaga. Phytopathology 56:624-626.

Xue, S., Cao, T., Howard, R. J., Hwang, S. F., and Strelkov, S. E. 2008. Isolation and variation in virulence of single-spore isolates of Plasmodiophora brassicae from Canada. Plant Dis. 92:456-462. 